\title{
An analysis of the relationship between ACE2 genetic polymorphisms and the severity of COVID- 19 disease in adult hospitalized patients - prospective observational study
}

Katarzyna Kotfis ( $\sim$ katarzyna.kotfis@pum.edu.pl)

Pomeranian Medical University

Jerzy Sieńko

Pomeranian Medical University

Izabela Marczak

Tufts Medical Center

Maciej Kotowski

Pomeranian Medical University

Anna Bogacz

Pomeranian Medical University

Karol Tejchman

Pomeranian Medical University

Magdalena Sieńko

Pomeranian Medical University

Research Article

Keywords:

Posted Date: February 28th, 2022

DOI: https://doi.org/10.21203/rs.3.rs-1337161/v1

License: (c) (1) This work is licensed under a Creative Commons Attribution 4.0 International License.

Read Full License 


\title{
An analysis of the relationship between ACE2 genetic polymorphisms and the severity of COVID-19 disease in adult hospitalized patients - prospective observational study
}

\author{
Jerzy Sieńko ${ }^{1}$, Izabela Marczak ${ }^{2}$, Maciej Kotowski ${ }^{1}$, Anna Bogacz ${ }^{3}$, Karol Tejchman ${ }^{1}$, \\ Magdalena Sieńko ${ }^{4}$, and Katarzyna Kotfis ${ }^{5, *}$ \\ ${ }^{1}$ Department of General Surgery and Transplantology, Pomeranian Medical University, 70-111, Szczecin, Poland \\ ${ }^{2}$ Department of Neurology, Tufts Medical Center, Boston, 02116, USA \\ ${ }^{3}$ Department of Stem Cells and Regenerative Medicine, Institute of Natural Fibers and Medicinal Plants, $62-064$ \\ Plewiska, Poland \\ ${ }^{4}$ Department of Pediatrics, Endocrinology,Diabetology, Metabolic Diseases and Cardiology, Pomeranian Medical \\ University, Szczecin, 71-252, Poland \\ ${ }^{5}$ Department of Anesthesiology, Intensive Therapy and Acute Intoxications, Pomeranian Medical University, \\ Szczecin, 70-111, Poland \\ *katarzyna.kotfis@pum.edu.pl
}

\begin{abstract}
The severe acute respiratory syndrome coronavirus 2 (SARS-CoV-2) first emerged in Hubei province of China in December 2019 and spread rapidly to other parts of the world, causing the coronavirus disease 2019 (COVID-19). The angiotensinconverting enzyme-2 (ACE2) is recognized as the host receptor used by the SARS-CoV-2 virus to enter its target cells. Recent studies suggest that ACE2 gene polymorphisms might be candidates for genetic susceptibility of SARS-CoV-2 infection. The aim of the study was to evaluate the influence of ACE2 polymorphisms on COVID-19 disease risk and severity. In our study we confirmed that there is a statistically significant increased risk of a more severe disease course of SARS-CoV-2 infection associated with the need for hospitalization in the intensive care unit for patients with specific polymorphisms of the ACE2 gene. The most significant correlation was found for variant ACE2 rs2285666 (AA allele, OR=2.12, $p=0.0189$ ) and ACE2 rs2074192 (TT allele, OR=2.05, $\mathrm{p}=0.0016$ ), and also for ACE2 rs4646174 (GG allele, OR=1.93, $\mathrm{p}=0.0016$ ), ACE2 rs4646156 (TT allele $\mathrm{OR}=1.71, \mathrm{p}=0.008$ ) and ACE2 rs2158083 (TT allele $\mathrm{OR}=1.84, \mathrm{p}=0.0025$ ). In conclusion, our findings identify that certain $\mathrm{ACE} 2$ polymorphisms impact the severity of COVID-19 disease independently of other well-known risk factors.
\end{abstract}

\section{Introduction}

Severe acute respiratory syndrome virus 2 (SARS-CoV-2) has caused a global pandemic of respiratory illness, called Coronavirus disease 2019 (COVID-19). The disease first emerged in December 2019 in Wuhan Province of China, and in a few months, the virus spread rapidly to other parts of the world ${ }^{1}$. Based on the data from a COVID-19 Dashboard created by the Center for Systems Science and Engineering (CSSE) at Johns Hopkins University, at the time of writing this manuscript, there were over 244 million confirmed cases of SARS-CoV-2 infections and nearly 5 million deaths around the world.

SARS-CoV-2 belongs to a family of Coronaviruses, relatively large, enveloped viruses with non-segmental, positive-stranded RNA molecules. Most coronaviruses, such as 229E, NL63, HKU1, and OC43, typically cause mild respiratory infection ${ }^{234}$. However, past decades have brought us three highly lethal members of coronaviruses, including SARS-CoV-1, MERS-CoV, and novel SARS-CoV-2 that causes Severe Acute Respiratory Syndrome (SARS), Middle East Respiratory Syndrome (MERS), and Coronavirus disease 19 (COVID-19), respectively. In contrast, the SARS-CoV-2 is much more contagious than SARS-CoV-1 or MERS-CoV ${ }^{56}$. However, SARS-CoV-1 and MERS-CoV have higher mortality rates than novel SARSCoV-2 ${ }^{7}$. Coronaviruses derived their name from the Latin word "crown", which refers to the crown-like appearance of the virus seen under the electron microscope ${ }^{8}$. SARS-CoV-2 has four structural proteins: membrane (M), envelope (E), spike (S), and nucleocapsid (N) proteins. Like SARS-CoV-1, the surface spike protein of SARS-CoV-2 is essential for viral attachment and entry into the host cell via the angiotensin converting enzyme 2 (ACE2) receptor ${ }^{910}$. ACE2 receptor is highly expressed in capillary-rich organs, including lungs, which may explain the predominance of respiratory symptoms of the COVID-19111213. ACE2 receptor is 
a crucial element of the renin-angiotensin system (RAS) responsible for regulating blood pressure. Its catalytic domain has over $40 \%$ identity to its homolog angiotensin-converting enzyme (ACE) ${ }^{1415}$. ACE is located in the lungs and converts inactive angiotensin I (AngI) to active angiotensin II (AngII) ${ }^{16}$. Angiotensin II binds to the AT1 receptor, causes vasoconstriction, and promotes inflammation or might enhance thrombosis via the AT4 receptor ${ }^{1718}$. In contrast, the ACE2 enzyme catalyzes the hydrolysis of angiotensin II to inactive angiotensin (1-7) (Ang (1-7)), which is a vasodilator agent with antihypertensive and anti-proliferation properties ${ }^{1920}$. Thus, the ACE2 is an important RAS negative factor and balances the ATII/Ang(1-7) ratio. The SARS-CoV-2 virus interacts with the ACE2 receptor and causes lower ACE2 availability and decreased breakdown of Ang II. Down-regulation of the ACE2 receptor might lead to severe acute respiratory failure with increased risk of cardiac injury and thrombosis. ${ }^{2122}$

ACE2 gene is located on chromosome Xp22 and contains 20 introns and 18 exons. Several studies confirmed that ACE2 polymorphisms are associated with a risk of primary hypertension and other cardiovascular diseases ${ }^{2324}$. The ACE2 variants associated with hypertension include rs2285666, rs879922, rs4646188, rs2106809, rs4240157, rs4830542, rs2158083, rs879922, rs1514283, rs2074192, rs4646155, rs4646176, rs4646174 and rs233575. While, left ventricular hypertrophy ACE2 polymorphisms include rs2106809, rs2074192, rs4646156, rs879922, rs4240157 and rs23357525. Cardiovascular diseases are well-known common co-morbidities of SARSCoV-2 infection that increase the risk of hospitalization and death. ${ }^{2627}$

Based on the recent literature, it seems obvious that the ACE2 gene polymorphisms might be crucial candidates in understanding the genetic susceptibility for SARS-CoV-2 infection. The aim of presented study was to evaluate the influence of ACE2 gene polymorphisms (rs2074192, rs2158083, rs2285666, rs4646156, rs4646174) on COVID-19 disease risk and severity.

\section{Results}

The study involved 188 individuals that were positive for the SARS-CoV-2 virus between September 2020 and May 2021. Patients were classified according to the severity of the infection: Group I (101 patients) with no or mild symptoms, such as fever, cough, muscle aches, loss of pain/taste, who were treated at home or in isolation treatment places; Group II (87 patients) with a severe course of coronavirus disease that required hospitalization in the intensive care unit and mechanical ventilation. The general demographic and clinical characteristics of groups I and II are present in Table 1. In Group II the percentage of men were higher in $(72.41 \%, \mathrm{p}<0.001)$, as was the BMI $(\mathrm{p}=0.001)$. The percentage of BMI categories in each group is present in Table 2. Additionally, there was a strong statistical significance between type 2 diabetes mellitus and severe SARS-CoV-2 infection outcome $(\mathrm{OR}=3.072, \mathrm{p}=0.0428)$. The odds risk $(\mathrm{OR})$ of being in Group II was 7.509 greater for patients with ischemic heart disease, but there was no statistical significance $(\mathrm{p}>0.05$ and $\mathrm{p}<0.1)$. Interestingly, hypothyroidism was associated with low significance to a milder course of COVID19 disease $(\mathrm{OR}=0.208, \mathrm{p}=0.006)$.

\section{Association of genetic variants of ACE2 gene with the risk of severe COVID-19 disease}

In our study, we confirmed that there is a statistically significant increased risk of a more severe disease outcome of SARS-CoV-2 infection associated with hospitalization in the intensive care unit for specific polymorphisms of the ACE2 gene (Table 3). The most significant correlation was found for variant ACE2 rs2285666 (AA allele, OR=2.12, p=0.019) and ACE2 rs2074192 (TT allele, $\mathrm{OR}=2.05, \mathrm{p}=0.002$ ), and for ACE2 rs4646174 (GG allele, OR=1.93, p=0.002), ACE2 rs4646156 (TT allele OR=1.71, $\mathrm{p}=0.008$ ) and ACE2 rs2158083 (TT allele $\mathrm{OR}=1.84, \mathrm{p}=0.003)($ Fig1).

\section{Association between ACE2 gene polymorphisms to general clinical characteristics and COVID-19 disease severity outcome}

We analyzed the correlation between ACE2 polymorphisms and different demographic and clinical characteristics in study subjects. We found statistically significant correlation between two ACE2 polymorphisms: rs4646156 (TT >AT, $\mathrm{p}=0.033025)$ and rs2158083 (TT>TC, p=0.029705), and higher BMI value. There was no significant correlation between ACE2 polymorphisms and age or diabetes mellitus type 1 or type 2 , hypertension, ischemic heart disease and other comorbidities.

\section{Discussion}

The aim of this prospective observational study was to report data regarding the possible association between ACE2 gene polymorphisms and disease severity in patients with SARS-CoV-2 infection. The results of our study demonstrate a statistically significant correlation between the ACE2 receptor gene rs2074192, rs2158083, rs2285666, rs4646156, rs4646174 polymorphisms, and the severity of COVID-19 in adult patients. To our best knowledge, we are the first authors that investigated these five ACE2 polymorphisms together in a wet lab fashion. The data found in other studies vary between these ACE2 gene variants. In our study, we found a strong correlation between ACE2 rs2074192 TT-genotype and poor outcome of patients with severe form of COVID-19. Our findings are consistent with a pilot study of Cafiero et al., who identified a higher frequency of T-allele of ACE2 rs2074192 in symptomatic vs. asymptomatic Italian patients ${ }^{28}$. Also, a recent study that included 1644 
French-Canadian and British patients showed that the T allele was associated with the severity of COVID-19 disease in obese smoking males ${ }^{29}$. Moreover, the rs2074192 T-allele is well known for its relation to cardiovascular risk and hypertension, common risk factors for COVID-19 disease ${ }^{30}$.

In a study by Möhlendick et al. the authors postulated that ACE2 rs2285666 GG-genotype is associated with severe COVID-19 outcome, whereas AA-genotype could have a "protective" role ${ }^{31}$. In contrast, we found opposite conclusions in our Polish population as rs2285666 AA-genotype increases the risk of being in the severe group of patients, and GA-genotype could be a "protective" variant. Also, our results contradict two different studies performed recently. The first study in which Gomez et al. compared 204 controls with 204 COVID-19 patients (137 non-severe and 67 severe) and reported no association between ACE2 rs2285666 polymorphism with COVID-19 severity in the Spanish population ${ }^{32}$. However, they found that ACE1 DD genotype, which was not investigated in our study, together with hypertension, hypercholesterolemia, and hypertensive male gender, were associated with poor outcomes of COVID-19 disease ${ }^{32}$. The second study in which Çelik et al. compared 155 COVID-19 patients via severity of the disease and found no relation of ACE2 rs2106809 and rs2285666 variants to the outcome of COVID-19 disease ${ }^{33}$. In contrast to the results of both above-mentioned studies, our patient's groups were more homogenous due to strict exclusion criteria, e.g., older age, severe obesity, and comorbidities, which was intentively used to optimize our findings. Our study revealed the correlation of rs4646156 and rs2158083 with higher BMI values. The ACE2 rs4646156 variant is known to be associated with diabetes mellitus type 2 and high total cholesterol (TC) levels, which are well known to be more frequent in obese patients ${ }^{3435}$. The severity of SARS-CoV-2 infection is well known to be associated with age, male sex, and some pre-existing diseases, including diabetes mellitus, hypertension, and other cardiovascular diseases. However, we found no significant correlation between ACE2 polymorphisms and age or diabetes mellitus type 1 or type 2, hypertension, ischemic heart disease, and other comorbidities. Sze et al. performed a meta-analysis, which included 18,728,893 patients from 50 studies, and found that individuals from Asian and Black ethnicities had a higher risk of SARS-CoV-2 infection than White individuals $^{36}$. Also, both the rate of ICU admission and death rate were higher in the Asian population. Although our study involved a Polish population of the study subjects, further studies comparing different ethnicities would be required.

In addition, some of the limitations of our study should be mentioned. Firstly, our sample size is limited, and further research in a larger group of patients is essential for validating our results. Secondly, the study subjects were limited to ethnicity and race of the Polish population. A future study in a more diverse patient population might explain some differences during COVID-19 in different ethnic, racial or geographic backgrounds. However, despite its limitations this study sheds an important light onto the association between the ACE2 gene polymorphisms and the severity of COVID-19.

In conclusion, we demonstrated the rs2074192, rs2158083, rs2285666, rs4646156, and rs4646174 polymorphisms of the ACE2 gene are associated with the severity of COVID-19 disease. Also, the ACE2 rs4646156 and rs2158083 variants were correlated with higher BMI values.

\section{Methods}

In this prospective observational study, we enrolled adult patients of both sexes, who were positive for the SARS-CoV-2 virus, confirmed with PCR standardized testing, who were symptomatic and who were not vaccinated. To minimize the possible interferences, such as older age or severe obesity, on the results we optimize groups via exclusion criteria for study subjects. The exclusion criteria were as follows: (1) age over 68 years; (2) BMI $>35 \mathrm{~kg} / \mathrm{m} 2$ except for relatively younger patients or patients without severe chronic illness (3) chronic kidney disease (CKD) treated with dialysis; (4) unknown and/or decompensated hypertension; (5) unknown and/or decompensated diabetes mellitus.

According to the manufacturer's protocol, genomic DNA was isolated from peripheral blood leukocytes using a commercially available PureLink $^{\mathrm{TM}}$ Genomic DNA Mini Kit (Thermo Fisher Scientific, Waltham, MA, USA). Briefly, $200 \mu 1$ of each blood sample was digested using proteinase $\mathrm{K}$ and RNase for $10 \mathrm{~min}$ at $55^{\circ} \mathrm{C}$. Afterward, the lysis buffer and ethanol were added, and the samples were loaded onto a silica-based membrane. After washing and elution steps, the DNA concentration was measured using Epoch Microplate Spectrophotometer (BioTek, Winooski, VT, United States). Analysis of the ACE2 (rs2074192, rs2158083, rs2285666, rs4646156, rs4646174) polymorphisms was performed by real-time PCR method using LightCycler®96 system (Roche Diagnostics). Sets of LightSNiPs (TibMolbiol, Germany) to determine ACE2 polymorphisms contained appropriate concentrations of specific primers and probes for the amplified fragment and were prepared according to the manufacturer's instructions. The PCR program was initiated at $95^{\circ} \mathrm{C}$ for $10 \mathrm{~min}$. Each PCR cycle comprised a denaturation step at $95^{\circ} \mathrm{C}$ for $10 \mathrm{~s}$, an annealing step at $60^{\circ} \mathrm{C}$ for $10 \mathrm{~s}$, and an elongation step at $72^{\circ} \mathrm{C}$ for $15 \mathrm{~s}$ ( 45 cycles). The final stage was the melting of products as a result of temperature rise to $95^{\circ} \mathrm{C}$. The reaction composition of a single sample was as follows: H2O - 6.7 ul, LightSNiP - 0.5 ul, LightCycler480 Genotyping Master - 1 ul , MgCl2 (25mM) - 0.8 ul, DNA (50 ng) - 1 ul. The analysis of the genotyping was based on the melting curve using LightCycler®96 Basic Software.

Statistical analysis was performed on raw data using Statistica 13 software package (TIBCO Software Inc., 3307 Hillview Avenue Palo Alto, CA 94304, USA). Polymorphisms in study groups were analyzed using frequency tables and Pearson's chi-squared, as well as nonparametric R Spearman, rang tests. Odds ratios and confidence intervals for polymorphisms and 
concomitant diseases were calculated using the creator of logistic regression. They all were analyzed as one-factor risk evaluation and in conjunction as a multifactor set. Continuous variables were analyzed with the use of t-Student test and Kruskal-Wallis ANOVA rang test. Statistical tests were chosen according to the input data and the cause-effect analytic questions. Statistical significance was set at $\mathrm{p}<0.05$.

All methods were carried out in accordance with relevant guidelines and regulations. All experimental protocols were approved by the Ethical Committee of the Pomeranian Medical University in Szczecin (Approval number: KB-0012/88/2020; approval date, June 22.06.2020 ). The study was conducted in accordance with the Helsinki Declaration (1975, revised 2000). We obtained approval from the Ethical Committee of the Pomeranian Medical University in Szczecin to perform our study without a written informed consent from all study subjects due to the observational nature of the study (Approval number KB-0012/88/2020; approval date, 22.03.2021).

\section{References}

1. Wu, F. et al. A new coronavirus associated with human respiratory disease in China. Nature 579, 265-269, DOI: 10.1038/s41586-020-2008-3 (2020).

2. Corman, V. M., Muth, D., Niemeyer, D. \& Drosten, C. Hosts and Sources of Endemic Human Coronaviruses. Adv. virus research 100, 163-188, DOI: 10.1016/bs.aivir.2018.01.001 (2018).

3. Gaunt, E. R., Hardie, A., Claas, E. C. J., Simmonds, P. \& Templeton, K. E. Epidemiology and clinical presentations of the four human coronaviruses 229E, HKU1, NL63, and OC43 detected over 3 years using a novel multiplex real-time PCR method. J. clinical microbiology 48, 2940-2947, DOI: 10.1128/JCM.00636-10 (2010).

4. Liu, D. X., Liang, J. Q. \& Fung, T. S. Human Coronavirus-229E, -OC43, -NL63, and -HKU1 (Coronaviridae)., DOI: 10.1016/B978-0-12-809633-8.21501-X (2021).

5. Hui, D. S. C. \& Zumla, A. Severe Acute Respiratory Syndrome: Historical, Epidemiologic, and Clinical Features. Infect. disease clinics North Am. 33, 869-889, DOI: 10.1016/j.idc.2019.07.001 (2019).

6. Nassar, M. S., Bakhrebah, M. A., Meo, S. A., Alsuabeyl, M. S. \& Zaher, W. A. Middle East Respiratory Syndrome Coronavirus (MERS-CoV) infection: epidemiology, pathogenesis and clinical characteristics. Eur. review for medical pharmacological sciences 22, 4956-4961, DOI: 10.26355/eurrev_201808_15635 (2018).

7. Cui, J., Li, F. \& Shi, Z.-L. Origin and evolution of pathogenic coronaviruses. Nat. reviews. Microbiol. 17, 181-192, DOI: 10.1038/s41579-018-0118-9 (2019).

8. Weiss, S. R. \& Navas-Martin, S. Coronavirus pathogenesis and the emerging pathogen severe acute respiratory syndrome coronavirus. Microbiol. molecular biology reviews : MMBR 69, 635-664, DOI: 10.1128/MMBR.69.4.635-664.2005 (2005).

9. Zhou, P. et al. A pneumonia outbreak associated with a new coronavirus of probable bat origin. Nature 579, 270-273, DOI: $10.1038 / \mathrm{s} 41586-020-2012-7$ (2020).

10. Scialo, F. et al. ACE2: The Major Cell Entry Receptor for SARS-CoV-2. Lung 198, 867-877, DOI: 10.1007/ s00408-020-00408-4 (2020).

11. Zou, X. et al. Single-cell RNA-seq data analysis on the receptor ACE2 expression reveals the potential risk of different human organs vulnerable to 2019-nCoV infection. Front. medicine 14, 185-192, DOI: 10.1007/s11684-020-0754-0 (2020).

12. Xu, H. et al. High expression of ACE2 receptor of 2019-nCoV on the epithelial cells of oral mucosa. Int. journal oral science 12, 8, DOI: 10.1038/s41368-020-0074-x (2020).

13. Hamming, I. et al. Tissue distribution of ACE2 protein, the functional receptor for SARS coronavirus. A first step in understanding SARS pathogenesis. The J. pathology 203, 631-637, DOI: 10.1002/path.1570 (2004).

14. Tipnis, S. R. et al. A human homolog of angiotensin-converting enzyme. Cloning and functional expression as a captopril-insensitive carboxypeptidase. The J. biological chemistry 275, 33238-33243, DOI: 10.1074/jbc.M002615200 (2000).

15. Donoghue, M. et al. A novel angiotensin-converting enzyme-related carboxypeptidase (ACE2) converts angiotensin I to angiotensin 1-9. Circ. research 87, E1-9, DOI: 10.1161/01.res.87.5.e1 (2000).

16. Voors, A. A. et al. Dual pathway for angiotensin II formation in human internal mammary arteries. Br. journal pharmacology 125, 1028-1032, DOI: 10.1038/sj.bjp.0702150 (1998). 
17. Ferrario, C. M., Trask, A. J. \& Jessup, J. A. Advances in biochemical and functional roles of angiotensin-converting enzyme 2 and angiotensin-(1-7) in regulation of cardiovascular function. Am. journal physiology. Hear. circulatory physiology 289, H2281-90, DOI: 10.1152/ajpheart.00618.2005 (2005).

18. Tikellis, C. \& Thomas, M. C. Angiotensin-Converting Enzyme 2 (ACE2) Is a Key Modulator of the Renin Angiotensin System in Health and Disease. Int. journal peptides 2012, 256294, DOI: 10.1155/2012/256294 (2012).

19. Sanchis-Gomar, F., Lavie, C. J., Perez-Quilis, C., Henry, B. M. \& Lippi, G. Angiotensin-Converting Enzyme 2 and Antihypertensives (Angiotensin Receptor Blockers and Angiotensin-Converting Enzyme Inhibitors) in Coronavirus Disease 2019. Mayo Clin. proceedings 95, 1222-1230, DOI: 10.1016/j.mayocp.2020.03.026 (2020).

20. Ferrario, C. M., Trask, A. J. \& Jessup, J. A. Advances in biochemical and functional roles of angiotensin-converting enzyme 2 and angiotensin-(1-7) in regulation of cardiovascular function. Am. journal physiology. Hear. circulatory physiology 289, H2281-90, DOI: 10.1152/ajpheart.00618.2005 (2005).

21. Zores, F. \& Rebeaud, M. E. COVID and the Renin-Angiotensin System: Are Hypertension or Its Treatments Deleterious? Front. cardiovascular medicine 7, 71, DOI: 10.3389/fcvm.2020.00071 (2020).

22. Sieńko, J. et al. COVID-19: The Influence of ACE Genotype and ACE-I and ARBs on the Course of SARS-CoV-2 Infection in Elderly Patients. Clin. interventions aging 15, 1231-1240, DOI: 10.2147/CIA.S261516 (2020).

23. Zhang, Q. et al. Association of angiotensin-converting enzyme 2 gene polymorphism and enzymatic activity with essential hypertension in different gender: A case-control study. Medicine 97, e12917, DOI: 10.1097/MD.0000000000012917 (2018).

24. Pan, Y. et al. Association of ACE2 polymorphisms with susceptibility to essential hypertension and dyslipidemia in Xinjiang, China. Lipids health disease 17, 241, DOI: 10.1186/s12944-018-0890-6 (2018).

25. Lieb, W. et al. Association of angiotensin-converting enzyme 2 (ACE2) gene polymorphisms with parameters of left ventricular hypertrophy in men. Results of the MONICA Augsburg echocardiographic substudy. J. molecular medicine (Berlin, Ger. 84, 88-96, DOI: 10.1007/s00109-005-0718-5 (2006).

26. Yang, J. et al. Prevalence of comorbidities and its effects in patients infected with SARS-CoV-2: a systematic review and meta-analysis. Int. journal infectious diseases : IJID : official publication Int. Soc. for Infect. Dis. 94, 91-95, DOI: 10.1016/j.ijid.2020.03.017 (2020).

27. Zheng, Y.-Y., Ma, Y.-T., Zhang, J.-Y. \& Xie, X. COVID-19 and the cardiovascular system. Nat. reviews. Cardiol. 17, 259-260, DOI: 10.1038/s41569-020-0360-5 (2020).

28. Cafiero, C. et al. Angiotensin System Polymorphisms' in SARS-CoV-2 Positive Patients: Assessment Between Symptomatic and Asymptomatic Patients: A Pilot Study. Pharmacogenomics personalized medicine 14, 621-629, DOI: 10.2147/PGPM.S303666 (2021).

29. Hamet, P. et al. SARS-CoV-2 Receptor ACE2 Gene Is Associated with Hypertension and Severity of COVID 19: Interaction with Sex, Obesity, and Smoking. Am. journal hypertension 34, 367-376, DOI: 10.1093/ajh/hpaa223 (2021).

30. Fan, Z. et al. Hypertension and hypertensive left ventricular hypertrophy are associated with ACE2 genetic polymorphism. Life sciences 225, 39-45, DOI: 10.1016/j.lfs.2019.03.059 (2019).

31. Möhlendick, B. et al. ACE2 polymorphism and susceptibility for SARS-CoV-2 infection and severity of COVID-19. Pharmacogenetics genomics 31, 165-171, DOI: 10.1097/FPC.0000000000000436 (2021).

32. Gómez, J. et al. Angiotensin-converting enzymes (ACE, ACE2) gene variants and COVID-19 outcome. Gene 762, 145102, DOI: 10.1016/j.gene.2020.145102 (2020).

33. Karakaş Çelik, S. et al. Polymorphisms of ACE (I/D) and ACE2 receptor gene (Rs2106809, Rs2285666) are not related to the clinical course of COVID-19: A case study. J. medical virology 93, 5947-5952, DOI: 10.1002/jmv.27160 (2021).

34. Liu, C. et al. ACE2 polymorphisms associated with cardiovascular risk in Uygurs with type 2 diabetes mellitus. Cardiovasc. diabetology 17, 127, DOI: 10.1186/s12933-018-0771-3 (2018).

35. Pan, Y. et al. Association of ACE2 polymorphisms with susceptibility to essential hypertension and dyslipidemia in Xinjiang, China. Lipids health disease 17, 241, DOI: 10.1186/s12944-018-0890-6 (2018).

36. Sze, S. et al. Ethnicity and clinical outcomes in COVID-19: A systematic review and meta-analysis. EClinicalMedicine 29, 100630, DOI: 10.1016/j.eclinm.2020.100630 (2020).

\section{Acknowledgements (not compulsory)}

None 


\section{Author contributions statement}

JS - designed the study, collected the data, analyzed the results, wrote the manuscript, provided funding and supervised project administration. IM - collected the data, analyzed the results, MK - designed the study, collected the data, analyzed the results, provided critical revision of the manuscript. AB analyzed and interpreted the patient data regarding genetic analysis, revised the manuscript critically. KT - performed statistical analysis, analyzed the results, provided graphics and critical revision of the manuscript. MS - analyzed the results and provided critical revision of the manuscript. KK - participated in the design of the study, collected the data, analyzed the results, made substantial contributions to the conception of the work and critically revised the manuscript. All the authors read and approved the final manuscript and agree to be accountable for all aspects of the work.

\section{Additional information}

Competing interests The authors declare that they have no competing interest.

Figure 1. Box plots of ACE2 polymorphisms
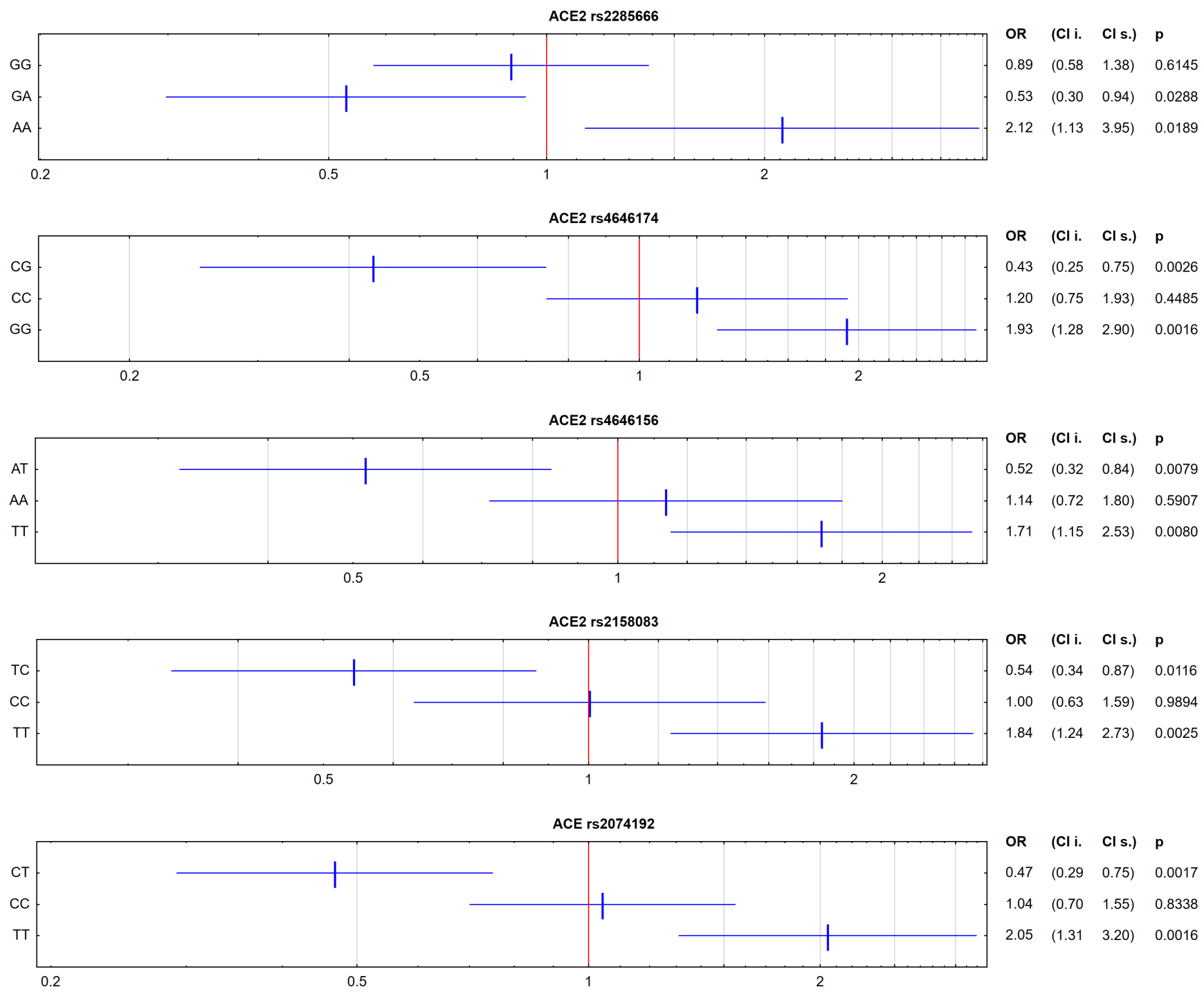

Legend: OR, odds ratio; $\mathrm{Cl}$ confidence interval; 
Table 1. General demographic and clinical characteristics

\begin{tabular}{|l|l|l|l|l|}
\hline Characteristics & Group I & Group II & OR (95\%) & P-value \\
\hline Age $($ mean \pm SD) & $54.17 \pm 3.86$ & $53.07 \pm 10.07$ & NA & 0.340 \\
\hline Female, $\mathrm{n}(\%)$ & $69(68.31)$ & $24(27.59)$ & $0.42[0.307-0.576]$ & $<0.001$ \\
\hline Male, $\mathrm{n}(\%)$ & $32(31.68)$ & $63(72.41)$ & $2.379[1.736-3.260]$ & $<0.001$ \\
\hline BMI (mean \pm SD) & $26.10 \pm 4.44$ & $28.23 \pm 3.97$ & NA & $<0.001$ \\
\hline Hypertension, $\mathrm{n}(\%)$ & $33(17.65)$ & $36(19.25)$ & $1.22[0.90-1.64]$ & 0.195 \\
\hline Diabetes mellitus type 2, n (\%) & $4(2.13)$ & $12(6.38)$ & $3.072[1.037-9.102]$ & 0.043 \\
\hline Hypothyroidism,, n (\%) & $16(15.8)$ & $4(4.56)$ & $0.208[0.068-0.638]$ & 0.006 \\
\hline
\end{tabular}

Legend: OR - odds ratio; $\mathrm{Cl}$ - confidence interval; NA - not applicable

Table 2. BMI categories in Group I and Group II

\begin{tabular}{|l|l|l|l|l|l|l|}
\hline BMI & Group & Number of Patients & Cumulative number & Percentage (\%) & Cumulative \% & $100 \%-\%$ \\
\hline BMI $<20$ & I & 6 & 6 & 5.940 & 5.941 & 100.000 \\
\hline BMI $\geq 20$ to $<25$ & I & 39 & 45 & 38.614 & 44.554 & 94.059 \\
\hline BMI $\geq 25$ to $<30$ & I & 42 & 87 & 41.584 & 86.139 & 55.445 \\
\hline BMI $\geq 30$ & I & 14 & 101 & 13.861 & 100.000 & 13.861 \\
\hline Undefined & I & 0 & 101 & 0 & 100.000 & 0.000 \\
\hline BMI $<20$ & II & 1 & 1 & 1.149 & 1.149 & 100.000 \\
\hline BMI $\geq 20$ to $<25$ & II & 17 & 18 & 19.540 & 20.690 & 98.851 \\
\hline BMI $\geq 25$ to $<30$ & II & 41 & 59 & 47.126 & 67.816 & 79.310 \\
\hline BMI $\geq 30$ & II & 28 & 87 & 32.184 & 100.000 & 32.184 \\
\hline Undefined & II & 0 & 87 & 0 & 100.000 & 0.000 \\
\hline
\end{tabular}

$B M I=w e i g h t(i n k g) / h e i g h t^{2}\left(\right.$ inm $\left.^{2}\right)$

Table 3. ACE2 polymorphism genotypes

\begin{tabular}{|c|c|c|c|c|}
\hline ACE2 polymorphism & Group I [n (\%)] & Group II [n (\%)] & OR (95\%) & P-value \\
\hline rs2285666 & & & & \\
AA & $8(4.26)$ & $16(8.51)$ & $2.12[1.13-3.95]$ & 0.019 \\
GG & $71(37.70)$ & $60(31.91)$ & $0.89[0.58-1.38]$ & 0.029 \\
GA & $22(11.70)$ & $11(5.85)$ & $0.53[0.30-0.94]$ & 0.614 \\
\hline rs4646174 & $44(23.40)$ & $57(30.32)$ & $1.93[1.28-2.90]$ & 0.002 \\
GG & $31(16.49)$ & $9(4.79)$ & $0.43[0.25-0.75]$ & 0.03 \\
CG & $26(13.83)$ & $21(11.17)$ & $1.20[0.75-1.93]$ & 0.449 \\
CC & $42(22.34)$ & $53(28.19)$ & $1.71[1.15-2.53]$ & 0.008 \\
rs4646156 & $34(18.09)$ & $13(6.91)$ & $0.52[0.32-0.84]$ & 0.008 \\
TT & $25(13.30)$ & $21(11.17)$ & $1.14[0.72-1.80]$ & 0.591 \\
AT & & & & \\
AA & $39(20.74)$ & $53(28.19)$ & $1.84[1.24-2.73]$ & 0.003 \\
\hline rs2158083 & $35(18.62)$ & $14(7.45)$ & $0.54[0.34-0.87]$ & 0.012 \\
TT & $27(14.36)$ & $20(10.64)$ & $1.00[0.63-1.59]$ & 0.989 \\
TC & & & & \\
CC & $20(10.64)$ & $34(18.09)$ & $2.05[1.31-3.20]$ & 0.002 \\
rs2074192 & $36(19.15)$ & $14(7.45)$ & $0.47[0.29-0.75]$ & 0.002 \\
TT & $45(23.94)$ & $39(20.74)$ & $1.04[0.70-1.55]$ & 0.834 \\
\hline CT & & & \\
CC & &
\end{tabular}

Legend: OR - odds ratio; $\mathrm{Cl}$ - confidence interval; 